\title{
Intermolecular Charge-Transfer-Induced Strong Optical Emission from Herringbone H-Aggregates
}

\author{
Qi Sun ${ }^{1}$, Jiajun Ren ${ }^{1}$, Tong Jiang ${ }^{1}$, Qian Peng ${ }^{2}$, Qi Ou ${ }^{1 *}$ and Zhigang Shuai ${ }^{*}$ \\ ${ }^{I}$ MOE Key Laboratory of Organic OptoElectronics and Molecular Engineering, Department of Chemistry, Tsinghua University, \\ Beijing 100084, P. R. China \\ ${ }^{2}$ School of Chemical Sciences, University of Chinese Academy of Sciences, Beijing, 100049, P. R. China
}

\begin{abstract}
Superior organic light-emitting transistors (OLETs) materials require two conventionally exclusive properties: strong luminescence and high charge mobilities. We propose a three-state model through localized diabatization to quantitative analyze excited state structures for various herringbone (HB) $\mathrm{H}$-aggregates and demonstrate that for some investigated systems, the low-lying intermolecular charge-transfer (CT) state couples with the bright Frenkel exciton (FE) and forms a dipole-allowed $\mathrm{S}_{1}$ that lies below the dark state, proceeding strong luminescence. Specifically, such conversion in luminescence properties occurs when the electron- and holetransfer integrals $\left(t_{\mathrm{e}}\right.$ and $\left.t_{\mathrm{h}}\right)$ are of the same sign and $t_{\mathrm{e}(\mathrm{h})}$ is notably larger than the excitonic coupling $(J)$, i.e., $t_{\mathrm{e}} \times t_{\mathrm{h}}>2 J^{2}$. This theoretical finding can not only explain and rationalize recent experimental results on DPA and dNaAnt, both with OLET property, but also unravel an exciting scenario where strong luminescence and high charge mobilities are compatible, which will considerably broaden the aperture of novel OLET design.
\end{abstract}

Key words: OLET, photoluminescence quantum yield, carrier mobility, H-aggregates.

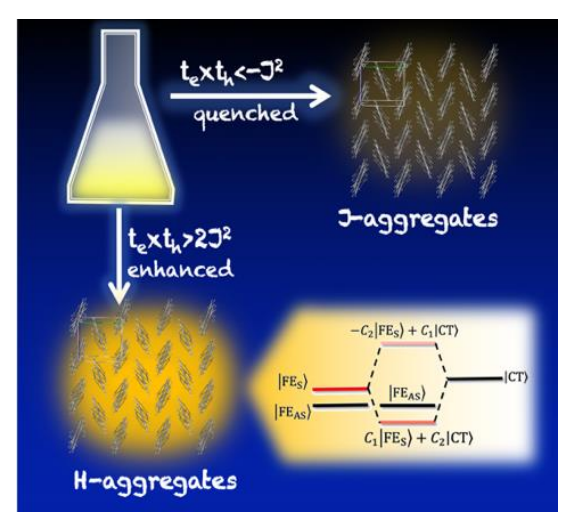

Table of Contents: By strongly coupling with the bright Frenkel exciton state, the intermolecular charge-transfer endows a transition dipole-allowed light-emitting state in $\mathrm{H}$ aggregates while guarantees high carrier mobilities, both of which are necessary in superior OLET materials. Theoretical findings as well as experimental observations confirm that strong emission and high mobility are indeed compatible in H-aggregates.

*Corresponding authors. Email addresses: qiou@tsinghua.edu.cn; zgshuai@ tsinghua.edu.cn. 


\section{Introduction}

Over the last two decades, rapid developments of organic charge transporting and light-emitting materials have proceeded the performance of organic light-emitting and display devices, and the promising potential of organic light-emitting transistors (OLETs) has been significantly accentuated. ${ }^{1-8}$ Compared with organic light-emitting diodes (OLEDs), OLETs combine the switching abilities of a transistor and can thus simplify the circuitry required for display applications. Owing to its capability of providing high current density and low optical loss at the electrodes, OLET also has great potential in realizing electrically pumped organic lasers. ${ }^{9-10}$ Nevertheless, superior OLET candidates require both strong photoluminescence and high charge mobility, which are normally considered as two contradictory properties and thus difficult to be simultaneously satisfied within one organic compound. ${ }^{3,7,11-12}$ Such contradiction arises from the fact that large transfer integrals, which are essential to high charge mobility, may introduce lowerlying intermolecular charge-transfer (CT) state to quench optical emission. Furthermore, the reported promising organic transistors to date are typically tight-packing $\mathrm{H}$-aggregates rather than J-aggregates, ${ }^{13}$ of which the emission process is prohibited within the traditional Kasha's exciton model (Figure 1). ${ }^{14}$ And it is generally conceived that in organic optoelectronic devices such as OLEDs, the carrier recombination is of Langevin's bimolecular process, ${ }^{15}$ namely, high mobility leads to fast nonradiative decay, which is detrimental to optical emission. Therefore, molecular design of OLET materials is yet full of challenges.

According to Kasha's exciton model (Figure 1), ${ }^{14}$ the lowest excited state of an H-aggregate dimer, with positive excitonic coupling $J$, is a transition dipole-forbidden antisymmetric Frenkel exciton (FE), and hence the emission of such $\mathrm{H}$-aggregate dimer is prohibited. For J-aggregate dimer, due to a negative exciton coupling, the lowest excited state becomes the transition dipoleallowed symmetric FE, of which the transition dipole moment is strengthened by a factor of $\sqrt{2}$ compared to that of the monomer. The emission of J-aggregate is thus enhanced upon aggregation, which corresponds to the origin of superradiance. ${ }^{16}$ While ubiquitously applied in plethora of organic crystalline systems, this classical Kasha picture relies on a 1D model and does not account for the effect of wavefunction overlaps, which is indeed essential to the photoluminescence as well as charge transport properties of the crystal. Some promising OLET crystalline materials, such as $\mathrm{DPA}^{17}$ and dNaAnt, ${ }^{18}$ attain enhanced luminescence upon aggregation, and are thus experimentally assigned as J-aggregates according to Kasha picture. Such assignment, however, lacks solid foundation due to the complexity of realistic 3D packing structure. In fact, theoretical studies have shown that $\mathrm{H}$ - and J-aggregate properties can simultaneously exist within one crystalline structure depending on the choice of the aggregate direction, and the one with stronger exciton coupling is usually experimentally observed. Moreover, such experimentally observed property can be altered by a number of factors such as the change of the temperature ${ }^{19}$ and the involvement of CT exciton (CTE) ${ }^{20-24}$ which would essentially adjust the energetic order of the excited states. While previous theoretical works mainly focus on $\pi$-stacking aggregates via means of parameterized model Hamiltonians and/or modeled aggregate systems, ${ }^{21-22,25}$ the luminescence mechanism of herringbone (HB) packing motif remains unclear, of which the hallmark is essentially different from its $\pi$-stacking counterpart. More importantly, on account of the aforementioned entangled factors, a quantitative description directly from $a b$ inito calculation for the luminescence mechanism of realistic crystalline aggregates is still encumbered.

Keeping these in mind, we focus on various HB aggregates (Scheme 1) in this work and propose a quantitative description of their photoluminescence mechanism through a diabatic Hamiltonian constructed from Boys localized diabatization method. ${ }^{26}$ The electronic structure properties of 
face-to-edge dimers in HB aggregates, which have shorter centroid distances compared to the slipped $\pi$ - $\pi$ dimers, are comprehensively explored. Our calculations evince that only one CTE lies closely to the FEs and presumably gets involved in the light-emitting process. A three-state diabatic Hamiltonian is then constructed, and analysis upon the diagonalization of the diabatic Hamiltonian underlines that the CTE may couple with the symmetric FE and forms a transition dipole-allowed $\mathrm{S}_{1}$ in H-aggregates if the electron and hole transfer integrals are of the same sign and their strength is noticeable larger than the exciton coupling. This hybrid $\mathrm{S}_{1}$ state lies below the dark state and ready for strong luminescence. An intermolecular CT-induced strong emission mechanism is hence unraveled, with the demonstration of an exciting scenario in which strong luminescence and high charge mobility are indeed compatible. We further investigate the role played by the packing configurations in the light-emitting properties and screen out the favored packing configurations for OLET materials. Altogether, by reconciling the two conventionally contradict factors in OLET, i.e., photoluminescence and charge mobility, our theoretical protocols presented here are expected to considerably broaden the aperture of novel OLET design in the future.

\section{H-AGGREGATE $(\boldsymbol{J}>0)$}

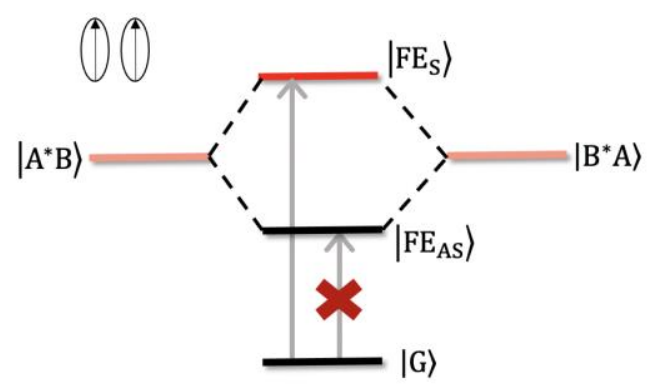

J-AGGREGATE $(\boldsymbol{J}<0)$

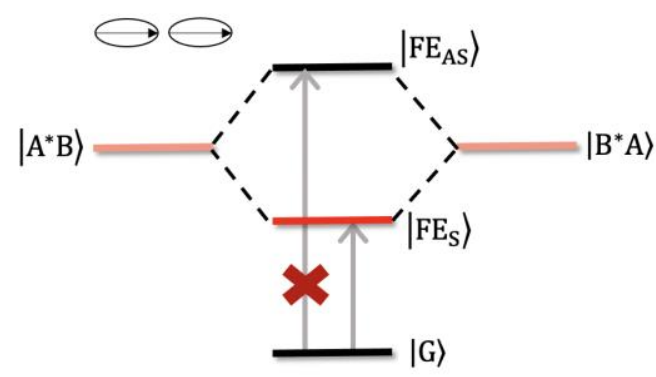

Figure 1. Schematic exciton coupling model for conventional Kasha $\mathbf{H}$ - and J-aggregate dimers. The transition dipole moments of each monomer point to the same direction, giving rise to the transition dipoleallowed symmetric $\mathrm{FE}\left(\left|\mathrm{FE}_{\mathrm{S}}\right\rangle\right)$ and transition dipole-forbidden antisymmetric $\mathrm{FE}\left(\left|\mathrm{FE}_{\mathrm{AS}}\right\rangle\right)$. The strength of the transition dipole moment is denoted by the saturation of the red color: $\left|\mathrm{FE}_{\mathrm{S}}\right\rangle$ has larger transition dipole moment compared to the excited states of each monomer $\left|A^{*} B\right\rangle$ and $\left|B^{*} A\right\rangle$, and black color corresponds to transition-dipole forbidden dark states.
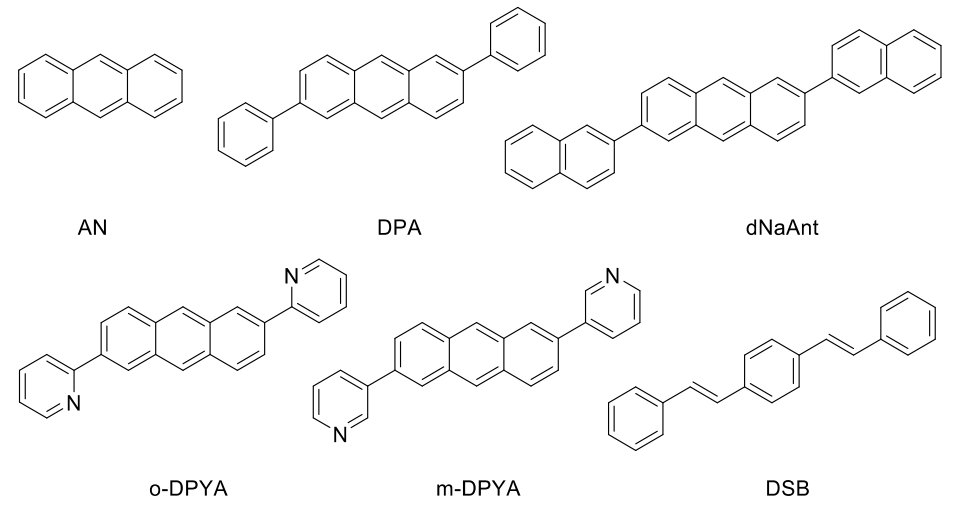

Scheme 1. Molecular structures of HB aggregates investigated in this work.

\section{Results}

A. Evaluation of the intermolecular interactions and CTE energies.

In two-dimensional HB lattice, four face-to-edge stacking dimers and two slipped $\pi$ - $\pi$ stacking dimers can be formed between one molecule and its nearest neighbors (Figure S1). The properties 
of two slipped $\pi-\pi$ stacking dimers are generally equivalent but those of four face-to-edge stacking dimers may slightly vary due to the marginally different centroid distances. We choose the faceto-edge stacking dimer with the shortest centroid distance and compare its properties with those of the slipped $\pi-\pi$ stacking dimer. As shown in Figure S2a, the intermolecular dispersion predicted by XSAPT + MBD in face-to-edge dimers is consistently stronger than that in slipped $\pi$ - $\pi$ stacking dimers for all investigated aggregates. Moreover, according to the point-dipole approximation, the exciton coupling $J$ is inversely proportional to the cube of the centroid distance between two monomers. Therefore, the face-to-edge stacking dimer also have stronger exciton coupling compared to slipped $\pi-\pi$ stacking dimers due to shorter centroid distances (as demonstrated in Table S1 for DSB). As a result, the face-to-edge stacking dimer, which is expected to exhibit more pronounced aggregation effect compared to the slipped $\pi-\pi$ counterpart due to stronger intermolecular interaction and exciton coupling, is employed to investigate the luminescence properties of the aggregation.

Within one face-to-edge stacking dimer, two types of CTE are formed, which can be denoted as $\mathrm{F}^{+} \mathrm{E}^{-}$(charge transferred from "face" part to "edge" part) and $\mathrm{F}^{-} \mathrm{E}^{+}$(charge transferred from "edge" part to "face" part). (See Figure S1 for the definitions of the "face" and "edge" parts.) The excitation energies of $\mathrm{F}^{-} \mathrm{E}^{+}$and $\mathrm{F}^{+} \mathrm{E}^{-}$for all investigated systems are shown in Table 1, together with the $\mathrm{S}_{1}$ excitation energies of monomers for comparison. It can be seen that $\mathrm{F}^{-} \mathrm{E}^{+}$is significantly lower in energy than $\mathrm{F}^{+} \mathrm{E}^{-}$and lies closely to the locally excited $\mathrm{S}_{1}$ state. Such energetic separation can be attributed to different intermolecular interactions inside these two CTEs. As shown in Figure S2b and Table S2, the overall attraction inside $\mathrm{F}^{-} \mathrm{E}^{+}$is stronger than that inside $\mathrm{F}^{+} \mathrm{E}^{-}$, which is mainly resulted from larger electrostatic and induction forces. Such stronger attraction stabilizes the energy of $\mathrm{F}^{-} \mathrm{E}^{+}$, making it energetically possible for $\mathrm{F}^{-} \mathrm{E}^{+}$(rather than $\mathrm{F}^{+} \mathrm{E}^{-}$) to couple with the locally excited states of the monomer.

Table 1. Excitation energy of the two types of CTEs and locally excited $\mathrm{S}_{1}$ state of the monomer in face-toedge dimers computed from Boys localized diabatization method at the level of $\omega B 97 \mathrm{X}-\mathrm{D} * / 6-31 \mathrm{G}(\mathrm{d})$. Numbers are shown in the unit of eV.

\begin{tabular}{cccc}
\hline Compound & $\boldsymbol{E}_{\mathbf{F}^{-} \mathbf{E}^{+}}$ & $\boldsymbol{E}_{\mathbf{F}^{+} \mathbf{E}^{-}}$ & $\boldsymbol{E}_{\mathbf{S}_{\mathbf{1}}}$ \\
\hline An & 4.05 & 4.79 & 3.64 \\
DPA & 3.38 & 4.16 & 3.32 \\
dNaAnt & 3.25 & 4.07 & 3.26 \\
o-DPYA & 3.49 & 4.20 & 3.41 \\
m-DPYA & 3.63 & 4.20 & 3.39 \\
DSB & 4.00 & 4.73 & $3.83 / 3.92^{\mathrm{a}}$ \\
\hline
\end{tabular}

${ }^{a}$ Two locally excited states have different energies. 


\section{B. Three-state Hamiltonian via Boys localized diabatization}

As discussed above, for HB aggregates investigated in this work, only one CTE $\left(\mathrm{F}^{-} \mathrm{E}^{+}\right)$is energetically favorable to couple with the locally excited state of each monomer (denoted as $\mathrm{F}^{*} \mathrm{E}$ and $\mathrm{FE}^{*}$ for consistency). Therefore, the electronic structure and photophysical properties can be comprehensively described via a three-state diabatic Hamiltonian. Within Boys localized diabatization, the diabatic Hamiltonian in the basis of $\left\{\left|\mathrm{F}^{*} \mathrm{E}\right\rangle ;\left|\mathrm{FE}^{*}\right\rangle ;\left|\mathrm{F}^{-} \mathrm{E}^{+}\right\rangle\right\}$is obtained for each system of interest, which is of the form:

$$
\widehat{\boldsymbol{H}}=\left[\begin{array}{ccc}
\boldsymbol{E} & \boldsymbol{J} & \boldsymbol{t}_{\mathrm{h}} \\
\boldsymbol{J} & \boldsymbol{E} & \boldsymbol{t}_{\mathrm{e}} \\
\boldsymbol{t}_{\mathrm{h}} & \boldsymbol{t}_{\mathrm{e}} & \boldsymbol{E}_{\mathrm{CT}}
\end{array}\right]
$$

where $E$ is the excitation energy of the locally excited state of each monomer; $E_{\mathrm{CT}}$ is the excitation energy of $\mathrm{F}^{-} \mathrm{E}^{+}$. A block-diagonalization is then applied to $\widehat{H}$ so that the Hamiltonian can be represented in the basis of $\left\{\left|\mathrm{FE}_{\mathrm{AS}}\right\rangle ;\left|\mathrm{FE}_{\mathrm{S}}\right\rangle ;\left|\mathrm{F}^{-} \mathrm{E}^{+}\right\rangle\right\}$(which is denoted as $\widehat{\mathcal{H}}$ ):

$$
\widehat{\mathcal{H}}=\left[\begin{array}{ccc}
E-J & 0 & \frac{\sqrt{2}}{2}\left(t_{\mathrm{h}}-t_{\mathrm{e}}\right) \\
0 & E+J & \frac{\sqrt{2}}{2}\left(t_{\mathrm{h}}+t_{\mathrm{e}}\right) \\
\frac{\sqrt{2}}{2}\left(t_{\mathrm{h}}-t_{e}\right) & \frac{\sqrt{2}}{2}\left(t_{\mathrm{h}}+t_{\mathrm{e}}\right) & E_{\mathrm{CT}}
\end{array}\right]
$$

To unravel the tacit luminescence mechanism of the aggregates, one needs to diagonalize $\widehat{\mathcal{H}}$ and explicitly analyze the resulting eigenstates as well as the eigenenergies, of which the expression is tediously long without any approximation and impractical to be employed for our analysis. Therefore, we perform the diagonalization within two simplified but practical scenarios, (i) $t_{\mathrm{h}} \approx t_{\mathrm{e}}=t$ and (ii) $t_{\mathrm{h}} \approx-t_{\mathrm{e}}=t$. For $t_{\mathrm{h}} \approx t_{\mathrm{e}}=t$, the eigenenergies are given by:

$$
\begin{gathered}
\boldsymbol{E}_{\mathbf{1}}=\boldsymbol{E}-\boldsymbol{J} \\
E_{2}=E+\frac{s+J-\sqrt{(s-J)^{2}+8 t^{2}}}{2} \\
E_{3}=E+\frac{s+J+\sqrt{(s-J)^{2}+8 t^{2}}}{2}
\end{gathered}
$$

where $s=E_{\mathrm{CT}}-E$; with the corresponding eigenstates:

$$
\Psi_{1}=\left|F_{A S}\right\rangle
$$

$$
\begin{gathered}
\Psi_{2}=\frac{1}{\sqrt{C^{2}+1}}\left(C\left|\mathrm{FE}_{\mathrm{S}}\right\rangle+\left|\mathrm{F}^{-} \mathrm{E}^{+}\right\rangle\right) \\
\Psi_{3}=\frac{1}{\sqrt{C^{2}+1}}\left(-\left|\mathrm{FE}_{\mathrm{S}}\right\rangle+C\left|\mathrm{~F}^{-} \mathrm{E}^{+}\right\rangle\right)
\end{gathered}
$$

where $C=E_{\mathrm{CT}}-E-J+\sqrt{\left(E_{\mathrm{CT}}-E-J\right)^{2}+8 t^{2}}$. It can be seen from Eqns. (6) to (8) that when $t_{\mathrm{h}} \approx t_{\mathrm{e}}=t$, CTE mainly couples with symmetric FE, i.e., the bright state with $\mu_{\mathrm{F}}$ and $\mu_{\mathrm{E}}$ pointing to the same direction, while the antisymmetric FE remains unchanged. Such expression holds for both $\mathrm{H}$-aggregate $(J>0)$ and $\mathrm{J}$-aggregate $(J<0)$. Recall that for $\mathrm{H}$-aggregate, the dark state $\left|\mathrm{FE}_{\mathrm{AS}}\right\rangle$ lies below $\left|\mathrm{FE}_{S}\right\rangle$, and therefore the emission process is expected to be prohibited without the mediation from other states. However, such traditional Kasha picture will break down when CTE 
gets involved. As schematically shown in Figure $2 \mathrm{a}$, if the coupling between $\left|\mathrm{FE}_{\mathrm{S}}\right\rangle$ and $\left|\mathrm{F}^{-} \mathrm{E}^{+}\right\rangle$is large enough, $\Psi_{2}$, which is a partially bright state, might be lower in energy than $\Psi_{1}$ and become the light-emitting state, and the emission process in such case will no longer be prohibited. Mathematically, the condition $E_{2}<E_{1}$ can be satisfied when $|t|>\sqrt{\left|J^{2}+J s\right|}$. Furthermore, when $\left|\mathrm{FE}_{\mathrm{S}}\right\rangle$ is lower in energy than $\left|\mathrm{F}^{-} \mathrm{E}^{+}\right\rangle$, i.e., $E+J\left\langle E_{\mathrm{CT}}\right.$, the contribution of $\left.\mid \mathrm{FE}_{\mathrm{S}}\right\rangle$ to $\Psi_{2}$ will exceed $50 \%$, and the resulting transition dipole moment of $\Psi_{2}$, the lowest excited (light-emitting) state, will be enhanced compared to that of the monomer. Such enhancement can be realized when $|t|>\sqrt{2}|J|$, which corresponds to the minimal requirement for $E_{2}<E_{1}$ and $E+J<E_{\mathrm{CT}}$. In other words, the H-aggregates may exhibit an enhanced emission process with red-shifted emission peak compared to the monomer (as schematically illustrated in Figure 2a), which conventionally appears in J-aggregates, under the condition that the hole and electron transfer integrals are of the same sign $\left(t_{\mathrm{h}} \approx t_{\mathrm{e}}=t\right)$ and their values are noticeably larger than the exciton coupling $(|t|>$ $\sqrt{2}|J|)$.

Conversely, for the second scenario, CTE mainly couples to the dark $\left|\mathrm{FE}_{\mathrm{AS}}\right\rangle$, while the bright $\left|\mathrm{FE}_{\mathrm{S}}\right\rangle$ remains unchanged, as demonstrated by the eigenstates of $\widehat{H}$ in the supplementary information. The lowest excited state of $\mathrm{H}$-aggregates in such case will always be dark since it is a linear combination of two transition dipole-forbidden states, $\left|\mathrm{FE}_{\mathrm{AS}}\right\rangle$ and $\left|\mathrm{F}^{-} \mathrm{E}^{+}\right\rangle$. Furthermore, for J-aggregates, in which $\left|\mathrm{FE}_{\mathrm{AS}}\right\rangle$ lies above $\left|\mathrm{FE}_{\mathrm{S}}\right\rangle$, the emission processes will be quenched if $t_{\mathrm{e}}$ or $t_{\mathrm{h}}$ is larger than $|J|$ and the hybrid dark state becomes the lowest excited state (as schematically illustrated in Figure 2b). Therefore, $t_{\mathrm{h}} \times t_{\mathrm{e}}<0$ is a presumably detrimental condition for strong luminescence upon aggregation. Note that for both two scenarios discussed above, if the absolute value of $t_{\mathrm{e}}$ and $t_{\mathrm{h}}$ is much small than that of the exciton coupling, CTE will negligibly couple with $\left|\mathrm{FE}_{\mathrm{S}}\right\rangle$ or $\left|\mathrm{FE}_{\mathrm{AS}}\right\rangle$ without shifting the lowest excited state, and the original behavior of $\mathrm{H}$ - or $\mathrm{J}$ aggregates will thus be conserved.

(a)

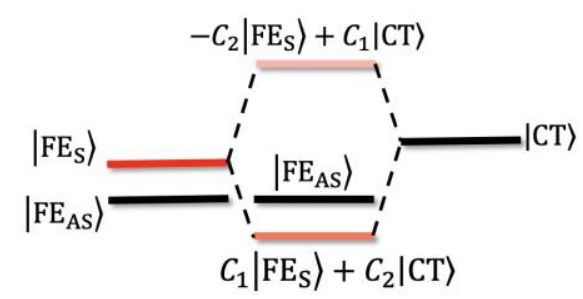

(b) SCENARIO II

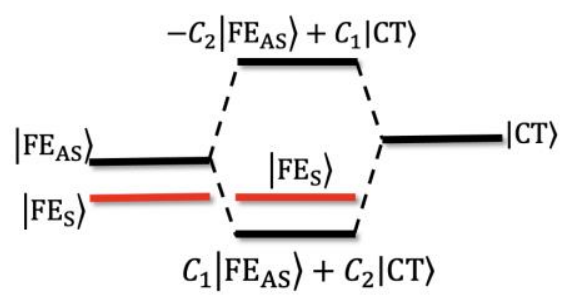

Figure 2. Schematic graph of the eigenstates of $\widehat{\mathcal{H}}$ for scenario $\mathrm{I}\left(t_{\mathrm{h}} \approx \boldsymbol{t}_{\mathrm{e}}=t\right)$ and scenario II $\left(t_{\mathrm{h}} \approx\right.$ $\left.-\boldsymbol{t}_{\mathbf{e}}=\boldsymbol{t}\right) \cdot C_{1}=C / \sqrt{C^{2}+1}, C_{2}=1 / \sqrt{C^{2}+1}$. The strength of the transition dipole moment is denoted by the saturation of the red color: state with more saturated red color has larger transition dipole moment, and black color corresponds to transition-dipole forbidden dark states.

To carry out quantitative analysis for HB aggregates investigated in this work, we choose DPA ${ }^{17}$ as a representative and its attachment-detachment densities of three localized diabatic states are shown in Figure 3. Explicit matrix elements of the diabatic Hamiltonian and the adiabatic-todiabatic rotation matrix as well as the attachment-detachment densities of the diabatic states for the rest of systems can be found in Table S3 and Figure S3. From the attachment-detachment densities, three diabatic states, $\left|\mathrm{F}^{*} \mathrm{E}\right\rangle,\left|\mathrm{FE}^{*}\right\rangle$, and $\left|\mathrm{F}^{-} \mathrm{E}^{+}\right\rangle$, are successfully constructed for each 
compound. The calculated exciton couplings, which corresponds to the value of the matrix element $\mathrm{H}_{12}$, are all positive, indicating that these face-to-edge dimers should be identified as $\mathrm{H}$-aggregates in conventional Kasha picture. The transfer integrals and exciton couplings of the investigated systems are also calculated via FMO method (Table S5), and the resulting values are in good agreement with those given by the localized diabatization method, which rationalizes the diabatization process.

Based on the aforementioned analysis, when $t_{\mathrm{h}} \times t_{\mathrm{e}}>0$, CTE couples to the bright symmetric FE; the photoluminescence will not be quenched if $|t|>\sqrt{\left|J^{2}+J s\right|}$ and will be enhanced if $|t|>$ $\sqrt{2}|J|$. From the calculated results, such condition is met in DPA, AN, and dNaAnt dimers, and the radiative decay rate $k_{\mathrm{r}}$ of the aggregates is thus expected to be increased compared to that of the isolated molecules. Specifically, the adiabatic $S_{1}$ and $S_{3}$ states of DPA are linear combinations of bright $\left|\mathrm{FE}_{\mathrm{S}}\right\rangle$ and $\left|\mathrm{F}^{-} \mathrm{E}^{+}\right\rangle$while $\mathrm{S}_{2}$ is mainly dark $\left|\mathrm{FE}_{\mathrm{AS}}\right\rangle$ according to the resulting rotation matrix in Table S3, which perfectly reproduce our first scenario, in which the hybrid $\Psi_{2}$ correspond to the light-emitting $\mathrm{S}_{1}$ state. Furthermore, the contribution of bright $\left|\mathrm{FE}_{S}\right\rangle$ to $\mathrm{S}_{1}$ of DPA is around $60 \%$, which yields an enhanced transition dipole moment upon aggregation. Note that even though DSB falls into the first scenario, its transfer integrals are significantly smaller compared to its exciton coupling, and mixed lower state of $\left|\mathrm{F}^{-} \mathrm{E}^{+}\right\rangle$and $\left|\mathrm{FE}_{\mathrm{S}}\right\rangle$ will still lie above $\left|\mathrm{FE}_{\mathrm{AS}}\right\rangle$, making it a traditional Kasha $\mathrm{H}$-aggregate that the radiative decay rate will be decreased upon aggregation. Contrarily, $t_{\mathrm{h}}$ and $t_{\mathrm{e}}$ are of the opposite sign in o-DPYA and m-DPYA, which fall into the second scenario where the CTE couples with dark FE, and the emission is expected to be prohibited. We compare our predicted change of $k_{\mathrm{r}}$ upon aggregation with experimental observations for these investigated systems in Table 2. It can be seen that the theoretical predictions are in perfect agreement with experiments, which rationalized our applied three-state model Hamiltonian. It should be noted that DPA has been previously assigned as J-aggregate due to the enhanced and red-shifted emission experimentally observed in crystal. ${ }^{17}$ This is not necessarily correct since the theoretically predicted exciton coupling for DPA is positive. The enhancement and red shift in the absorption process upon aggregation are more possibly contributed from the mediation of the CTE, which strongly couples to the bright FE and forms a transition dipole-allowed light-emitting state.

Table 2. Exciton couplings $(J)$ and transfer integrals $\left(t_{\mathrm{h}}, t_{\mathrm{e}}\right)$ evaluated via Boys localized diabatization method for all investigated systems. Numbers are shown in the unit of meV. The theoretical predicted and experiment observed change of $k_{\mathrm{r}}$ upon aggregation are also listed.

\begin{tabular}{cccccc}
\hline Compound & $\boldsymbol{J}$ & $\boldsymbol{t}_{\mathbf{h}}$ & $\boldsymbol{t}_{\mathbf{e}}$ & $\boldsymbol{k}_{\mathbf{r}}($ Theo $)$ & $\boldsymbol{k}_{\mathbf{r}}($ Exp $)$ \\
\hline $\mathrm{An}^{35}$ & 8 & 32 & 69 & $\uparrow$ & $\uparrow$ \\
$\mathrm{DPA}^{17}$ & 12 & 76 & 62 & $\uparrow$ & $\uparrow$ \\
$\mathrm{dNaAnt}^{18}$ & 26 & 60 & 66 & $\uparrow$ & $\uparrow$ \\
o-DPYA $^{36}$ & 7 & 93 & -36 & $\downarrow$ & $\downarrow$ \\
$\mathrm{m}^{-D P Y A}$ & & & & \\
$\mathrm{DSB}^{36}$ & 6 & 78 & -41 & $\downarrow$ & $\downarrow$ \\
\hline
\end{tabular}


Our three-state model Hamiltonian can be further validated via the QM/MM excited state calculations of which one face-to-edge dimer is treated as the QM part. From Table S4, the calculated transition dipole moments of AN, DPA, and dNaAnt exhibit comparable or even larger magnitude compared to their monomer counterparts, while those of o-DPYA, m-DPYA and DSB are considerably decreased upon aggregation. These QM/MM results are consistent with what we have drawn from the three-state diabatic Hamiltonian. The significance and rationality of the diabatic Hamiltonian not only rely on the resulting correct electronic structure properties, but indeed on the unraveled insights of the CT-enhanced strong emission mechanism of H-aggregates, which cannot be disclosed by the direct QM/MM calculation.

\section{The role of CTE with different orientation and displacements}

From the above discussion, the relative sign of the electron and hole transfer integrals is essential to the luminescence properties of the aggregates, and the CTE have completely contrary effect to the emission process under depending on the sign of $t_{\mathrm{h}} \times t_{\mathrm{e}}$. While the CTE is beneficial to the emission processes in $\mathrm{H}$-aggregates when $t_{\mathrm{h}} \times t_{\mathrm{e}}>0$, it will presumably quench the emission, even in J-aggregates if $t_{\mathrm{h}} \times t_{\mathrm{e}}<0$ and the absolute value of $t_{\mathrm{h}}$ and $t_{\mathrm{e}}$ is significantly larger than that of the exciton coupling. Therefore, it is of significant importance to explore the relationship between the explicit crystal structure and the relative sign of $t_{\mathrm{h}}$ and $t_{\mathrm{e}}$ so as to quickly screen out the light-emitting favored systems.

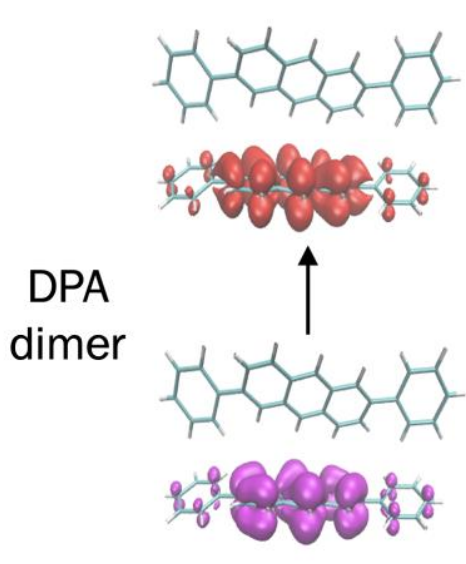

$F^{*} \mathrm{E}: 3.32 \mathrm{eV}$
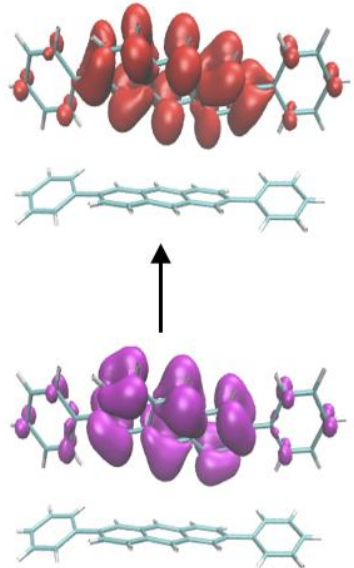

$\mathrm{FE}^{*}: 3.32 \mathrm{eV}$

$23.11 \%$
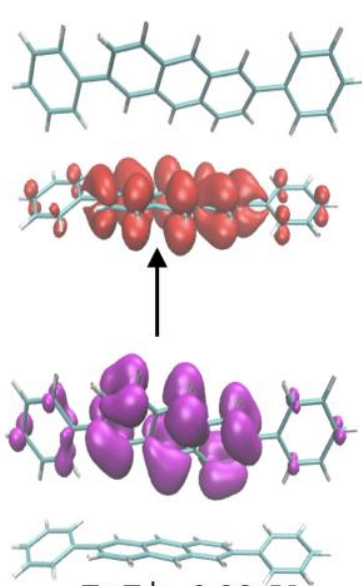

$\mathrm{F}^{-} \mathrm{E}^{+}: 3.38 \mathrm{eV}$

$37.01 \%$

Figure 3. Attachment-detachment densities and excitation energies of the resulting diabatic states, $\mathbf{F E}^{*}, \mathbf{F}^{*} \mathbf{E}$, and $\mathbf{F}^{-} \mathbf{E}^{+}$of DPA. The percentages listed below the excitation energy are the contribution of the corresponding diabatic state to the adiabatic $S_{1}$ state of DPA.

Taking DPA as an example, in Figure $4 \mathrm{a}$ and $\mathrm{b}$, we plot two possible configurations of the dimer, depending on the orientation of the twofold screw rotation axis $\hat{S}_{2}$ (plotted in blue in Figure 4 a and b). In anthracene derivatives studied in this work, An, DPA, and dNaAnt are eclipsed dimers, of which $t_{\mathrm{h}}$ and $t_{\mathrm{e}}$ are of the same sign, while the rest two systems are staggered dimers, of which $t_{\mathrm{h}}$ and $t_{\mathrm{e}}$ have opposite sign. The eclipsed structures are seemingly profitable to the photoluminescence. Nevertheless, caution has to been taken when exploring the dependence of the transfer integrals to the crystals structure, as previous studies have demonstrated that the transfer 
integrals are also very sensitive to the shape of the frontiers orbitals as well as displacement of the short and long axis, and sign of the transfer integral might be changed depending on the shape of the frontier orbitals and displacement between two monomers. ${ }^{37}$

To make a more solid connection between the crystal structure and the transfer integrals, we take DPA as a representative, constructing the eclipsed and staggered configurations of its face-toedge dimers, and slip one of the monomers along the short and long axes (Figure 4a and b) and calculate the transfer integral at various slipping coordinates. The rationality of such modeled packing structure lies in the fact that the frontier orbitals of these five anthracene derivates mainly localize on the anthracene moiety with almost identical node contributions (Figure S4). Note that the direction orthogonal to the defined short and long axes will only influence the strength of the transfer integrals and the exciton coupling but not their relative signs, we therefore fix the distance between two monomers along that direction. The displacement along the long axis is set to be $1.00 \AA \sim 3.00 \AA$, while the one along the short axis is set to be $-0.22 \AA \sim 0.08 \AA$ according to the practical packing structure of anthracene derivatives studied in this work. As shown in Figure 4c and $\mathrm{d}$, nearly opposite behaviors of the relative sign of $t_{\mathrm{h}}$ and $t_{\mathrm{e}}$ are found in eclipsed and staggered dimers within the displacement region while the exciton coupling is consistently positive (Figure $4 \mathrm{e}$ and $\mathrm{f}$ ), indicating the contrary role played by CTE in these two packing configurations. Moreover, the sign of $t_{\mathrm{h}} \times t_{\mathrm{e}}$ remain unchanged in both eclipsed dimers or staggered dimers when we change the herringbone angle from $40^{\circ}$ to $60^{\circ}$ by rotating one of the molecules, which covers the range of the herringbone angels for all investigated systems (Figure S5). Altogether, these finding reinforce that when a low-lying CTE couples with FEs in HB H-aggregates of anthracene derivatives, the eclipsed stacking configurations tend to exhibit enhanced $k_{\mathrm{r}}$ in crystals due to the fact that the sign of $t_{\mathrm{h}} \times t_{\mathrm{e}}$ is generally positive for a wide range of modeled packing structures, while $t_{\mathrm{h}}$ and $t_{\mathrm{e}}$ tend to be opposite and/or small in staggered stacking configurations, which are therefore unfavorable packing configurations for light-emitting crystals and the design of OLET materials. Modeled packing structures with larger displacements have also been investigated in Figure $\mathrm{S} 6$, and the resulting signs of $t_{\mathrm{h}} \times t_{\mathrm{e}}$ and the exciton coupling can be employed as a quick predictor to evaluate the photoluminescence and charge transport properties for a variety of anthracene derivatives. 
(a)

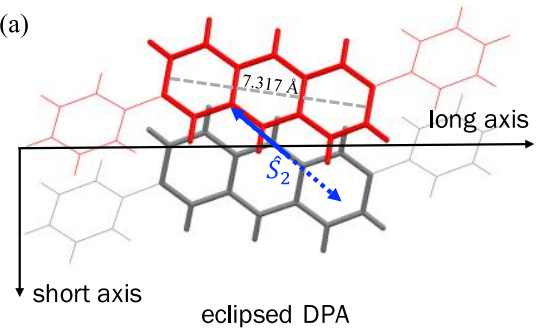

(c)

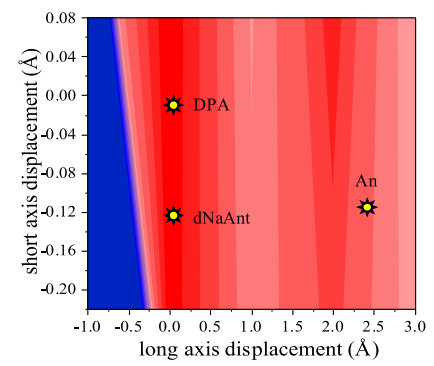

(e)

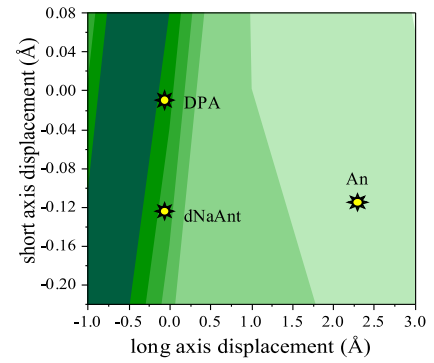

(b)

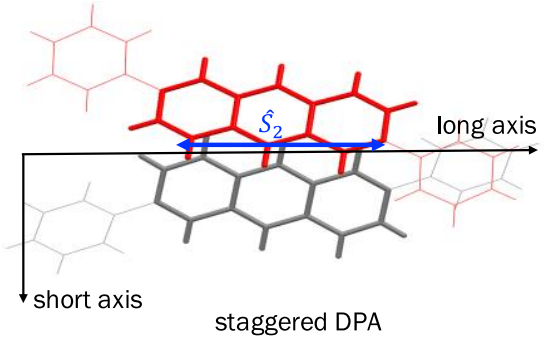

(d)

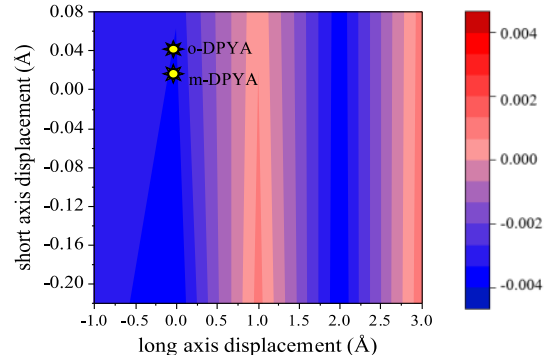

(f)

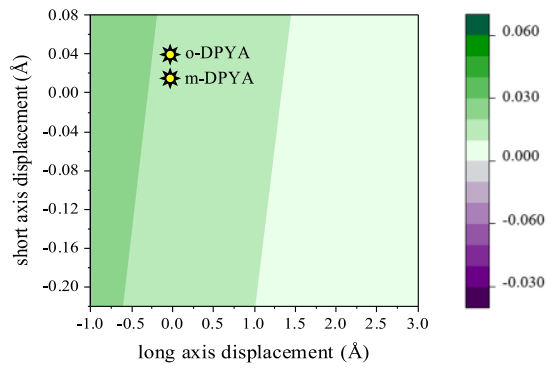

Figure 4. Two packing motifs and the influence of the packing motif to transfer integral and exciton coupling. (a) and (b) correspond to the structure of eclipsed and staggered DPA face-to-edge dimers; (c) and (e) correspond to the calculated $t_{\mathrm{h}} \times t_{\mathrm{e}}$ (in unit of $\mathrm{eV}^{2}$ ) and $J$ (in unit of eV) of the eclipsed DPA dimer with respect to the displacements along the short and long axes; (d) and (f) correspond to those of the staggered DPA dimer.

\section{Discussion and conclusion}

To conclude, we have proposed a novel scenario to achieve high luminescence as well as presumably high charge mobilities for H-type aggregates with the aid of intermolecular charge transfer. We have comprehensively and quantitatively analyzed the nature of the lowest-lying excited state in six HB H-aggregates via Boys localized diabatization and other sophisticated electronic structure methods. In our theoretical modelling, face-to-edge dimers inside the aggregates are chosen as the representative chromophore due to their stronger intermolecular interactions and larger exciton coupling compared to their $\pi-\pi$ counterparts. Based on the analysis, two types of CTEs $\left(\mathrm{F}^{-} \mathrm{E}^{+}\right.$and $\left.\mathrm{F}^{+} \mathrm{E}^{-}\right)$in face-to-edge dimers are energetically well-separated, which is resulted from the stronger electrostatic and induction forces inside $\mathrm{F}^{-} \mathrm{E}^{+}$that stabilize its energy and makes it lie closely to the locally excited states of each monomer (while the energy of $\mathrm{F}^{+} \mathrm{E}^{-}$is much higher). The three-state diabatic Hamiltonian has then been constructed via Boys localized diabatization to quantitatively unravel the emission mechanism of the investigated $\mathrm{H}-$ aggregates. When the electron and hole transfer integrals are of the same sign $\left(t_{\mathrm{h}} \approx t_{\mathrm{e}}=t\right)$ and their absolute values are noticeably larger than that of the exciton coupling $(|t|>\sqrt{2}|J|)$, the 
intermolecular CTE strongly couples with the bright Frenkel exciton (FE) and forms a dipoleallowed $\mathrm{S}_{1}$, which lies below the dark state and ready for strong luminescence. Such enhanced luminescence behavior, which traditionally recognized as the hallmark of the J-aggregates, are thus attained in $\mathrm{H}$-aggregates with considerable transfer integrals. Conversely, if $t_{\mathrm{h}} \approx-t_{\mathrm{e}}=t$, the emission enhancement will not occur in H-aggregates since CTE couples with the dark FE. When such coupling is large enough, the light-emitting state might become a dark state and emission in J-aggregates would be prohibited. Therefore, systems with $t_{\mathrm{h}} \times t_{\mathrm{e}}>0$ are advantageous OLET candidates of which the photoluminescence and charge transport are indeed compatible.

Two possible stacking confirmations of the face-to-edge dimers in HB aggregates, i.e., eclipsed and staggered, are then explored to investigate the relationship between the sign of $t_{\mathrm{h}} \times t_{\mathrm{e}}$ and the crystal packing structure. By properly slipping one of the monomers along the short and long axes, we have explored the sign of $t_{\mathrm{h}} \times t_{\mathrm{e}}$ within the practical packing range of anthracene derivatives and found out that the eclipsed configuration almost always has positive $t_{\mathrm{h}} \times t_{\mathrm{e}}$ and thus becomes a favored configuration for OLETs. Contrarily, the staggered configuration tends to have negative and/or negligible $t_{\mathrm{h}} \times t_{\mathrm{e}}$, which makes it a less preferred configuration to simultaneously realize strong luminescence and high charge mobility for systems investigated in this work and other similarly packed anthracene derivatives.

In summary, the tacit mechanism of the intermolecular CT-enhanced strong emission in $\mathrm{HB} \mathrm{H}$ aggregates has been explicitly disclosed in this work, which demonstrates an OLET-favored scenario where the photoluminescence and charge mobility are not mutually exclusive but compatible with each other. Looking forward, our theoretical protocol presented here would promise more advanced OLET materials with brighter emission and higher mobilities in the future.

\section{Methods and computational details}

To quantitatively construct the diabatic Hamiltonian for the investigated systems, we extend the previously reported Boys localized diabatization method, ${ }^{26}$ which is based on Tamm-Dancoff approximation, into the full TDDFT framework, i.e., within random phase approximation (RPA). The basic premise behind localized diabatization is to generate an adiabatic-to-diabatic rotation matrix by maximizing a localization function. Analogous to the well-known Boys orbital localization algorithm, Boys localized diabatization method constructs the diabatic states by maximizing the distance between the centroids of different states, and the corresponding localization function is given as

$$
f_{\text {Boys }}(\mathrm{U})=f_{\text {Boys }}\left(\left\{\left|\Xi_{I}^{\mathrm{LD}}\right\rangle\right\}\right)=\sum_{I J=1}^{N}\left|\left\langle\Xi_{I}^{\mathrm{LD}}|\vec{\mu}| \Xi_{I}^{\mathrm{LD}}\right\rangle-\left\langle\Xi_{J}^{\mathrm{LD}}|\vec{\mu}| \Xi_{J}^{\mathrm{LD}}\right\rangle\right|^{2}
$$

where $\left\{\left|\Xi_{I}^{L D}\right\rangle\right\}$ refers to a set of localized diabatic states constructed from a rotation of a set of adiabatic states $\left\{\left|\Psi_{J}\right\rangle\right\}$, and $\mathbf{U}$ is the corresponding rotation matrix, i.e.,

$$
\left|\Xi_{I}^{\mathrm{LD}}\right\rangle=\sum_{I, J=1}^{N} U_{I J}\left|\Psi_{J}\right\rangle
$$

The dipole moment of each localized diabatic states can then be expressed as a linear combination of the dipole moment of each adiabatic state and the transition dipole moment between different adiabatic states. Under the atomic orbital (AO) basis, the latter is written as

$$
\left\langle\Psi_{I}|\vec{\mu}| \Psi_{J}\right\rangle=\sum_{\mu v} M_{\mu v} D_{\mu v}^{I J}
$$


where indices $\mu$ and $v$ denote AOs; $M_{\mu \nu}$ the matrix element of the dipole operator in AO basis set; $D_{\mu \nu}^{I J}$ is the generalized difference density matrix between adiabatic states $I$ and $J$. Within RPA, $D_{\mu \nu}^{I J}$ is expressed as

$$
D_{\mu v}^{I J}=\sum_{i a b} C_{\mu a}\left(X_{i}^{I a} X_{i}^{J b}+Y_{i}^{I a} Y_{i}^{J b}\right) C_{v b}-\sum_{i j a} C_{\mu i}\left(X_{i}^{I a} X_{j}^{J a}+Y_{i}^{I a} Y_{j}^{J a}\right) C_{v j}
$$

where indices $i$ and $j$ denote occupied molecular orbitals (MOs), and $a$ and $b$ denotes virtual MOs. $\mathbf{X}^{I}$ and $\mathbf{Y}^{I}$ are excitation and de-excitation coefficients of adiabatic states $I$, and $\mathbf{C}$ is the MO coefficients matrix. Once the localization function $f_{\text {Boys }}(\mathbf{U})$ is maximized, the corresponding $\mathbf{U}$ and the localized diabatic states are determined. By properly choosing the adiabatic states of a dimer inside an aggregate system, one should be able to obtain multiple excited states with pure local excitation (LE) or intermolecular CT transition characters.

Practically, we plot the attachment-detachment densities ${ }^{27}$ of each localized diabatic state to justify its transition character and the effectivity of the localized diabatization. If the diabatization is successfully justified, i.e., the diabatic states are of expected pure transition characters, the resulting diabatic Hamiltonian will be applied for the analysis of the photoluminescence mechanism of the aggregates. Note that the off-diagonal elements of the diabatic Hamiltonian correspond to either the exciton coupling between two LE states or the electron coupling between the LE and CT states. Both of the exciton coupling and the electron coupling can be alternatively evaluated via the fragment molecular orbital (FMO) based method. If $\mathrm{AB}^{*}$ and $\mathrm{A}^{*} \mathrm{~B}$ are dominated by the transition between the frontier orbitals on each monomer, and CT corresponds to an intermolecular transition from the highest occupied molecular orbital (HOMO) of one monomer to the lowest unoccupied molecular orbital (LUMO) of the other monomer, the electron coupling can be expressed as

$$
\begin{gathered}
\left\langle\mathbf{C T}|\widehat{\boldsymbol{H}}| \mathbf{A B} \mathbf{B}^{*}\right\rangle \approx \boldsymbol{t}_{\mathbf{h}} \text { or } \boldsymbol{t}_{\mathbf{e}} \\
\left\langle\mathrm{CT}|\widehat{H}| \mathrm{A}^{*} \mathrm{~B}\right\rangle \approx t_{\mathrm{e}} \text { or } t_{\mathrm{h}} \\
t_{\mathrm{h}} \equiv-\left\langle\psi_{\text {Номо }}^{\mathrm{A}}|\hat{F}| \psi_{\text {Номо }}^{\mathrm{B}}\right\rangle, t_{\mathrm{e}} \equiv\left\langle\psi_{\mathrm{LUMO}}^{\mathrm{A}}|\hat{F}| \psi_{\mathrm{LUMO}}^{\mathrm{B}}\right\rangle
\end{gathered}
$$

where $t_{h}$ and $t_{e}$ are transfer integrals between the HOMO of the two monomers, i.e., $\psi_{\text {номо }}^{\mathrm{A}}$ and $\psi_{\text {HOMO }}^{\mathrm{B}}$, and the LUMO of the two monomers, i.e., $\psi_{\mathrm{LUMO}}^{\mathrm{A}}$ and $\psi_{\mathrm{LUMO}}^{\mathrm{B}}$, respectively. As shown in Figure S4, for all investigated systems in this work, the $\mathrm{S}_{1}$ states of each monomer are dominated by the HOMO to LUMO transition, and therefore Eqns. (13) to (15) hold for these systems.

Unless otherwise specified, all excited state calculations are carried out via TDDFT with optimal-tuned $\omega$ B97X-D (denoted as $\omega$ B97X-D*) functional and 6-31G(d) basis set. Following the procedure proposed in the reference, ${ }^{28}$ we tune the range-separate parameter $\omega$ based on the B3LYP/6-31G(d) optimized ground state structure of each isolated molecule, and explicit values of $\omega$ are listed in Table S1. Energetics of intermolecular CT states and LE excited states are evaluated via Boys localized diabatization method, as well as their coupling elements. Extended symmetry-adapted perturbation theory (XSAPT) and many-body dispersion (MBD) method ${ }^{29}$ (XSAPT+MBD) is applied at the level of CAM-B3LYP/def2-tzpp to decipher the intermolecular interactions, which is sensitive to the choice of the basis set. All of the above calculations are performed in a developmentary version of quantum chemistry package Q-chem. ${ }^{30}$ Calculations of transfer integrals $\left(t_{\mathrm{e}(\mathrm{h})}\right)$ and exciton couplings $(J)$, which can be evaluated via Boys localized diabatization method as discussed above, are also carried out via FMO based method in PySCF package $^{31}$ to efficiently determine $t_{\mathrm{e}(\mathrm{h})}$ and $J$, and further validate the rationality of the localized 
diabatization method. Finally, quantum mechanics and molecular mechanics (QM/MM) method with ONIOM model ${ }^{32}$ is utilized to compute the electronic structure properties of the excited state $\left(\mathrm{S}_{1}\right)$ for each $\mathrm{H}$-aggregate dimer so that our theoretical model can be justified from another perspective. Specifically, for each investigated system, a $3 \times 3 \times 3$ cluster are carved out of the crystal structure identified via X-ray diffraction (XRD), and the central two molecules are treated as QM part with $\omega \mathrm{B} 97 \mathrm{X}-\mathrm{D} * / 6-31 \mathrm{G}(\mathrm{d})$ while the surroundings are treated as MM part with the universal force field (UFF). The QM/MM calculations are performed in Gaussian16 program package. ${ }^{33}$

\section{Acknowledgements}

This work was supported by the National Natural Science Foundation of China Grant Nos. 21788102 and 22003030, as well as by the Ministry of Science and Technology of China through the National Key R\&D Plan, Grant No. 2017YFA0204501. QO is also funded by China Postdoctoral Science Foundation Grant No. 2020M670280. QO and JR are also supported by the Shuimu Tsinghua Scholar Program.

\section{Author contributions}

ZS and QO conceived the project. QS carried out the calculations. QP, TJ, and JR helped with the calculation process and provided inspiring suggestions for improvement. All authors contributed to data analysis and writing the paper.

\section{Competing interests}

The authors declare no competing interests.

\section{Reference}

1. Capelli, R.; Toffanin, S.; Generali, G.; Usta, H.; Facchetti, A.; Muccini, M., Organic lightemitting transistors with an efficiency that outperforms the equivalent light-emitting diodes. Nat Mater 2010, 9 (6), 496-503.

2. Zhang, C.; Chen, P.; Hu, W., Organic Light-Emitting Transistors: Materials, Device Configurations, and Operations. Small 2016, 12 (10), 1252-94.

3. Yuan, D.; Sharapov, V.; Liu, X.; Yu, L., Design of High-Performance Organic LightEmitting Transistors. ACS Omega 2020, 5 (1), 68-74.

4. Park, S. K.; Kim, J. H.; Ohto, T.; Yamada, R.; Jones, A. O. F.; Whang, D. R.; Cho, I.; Oh, S.; Hong, S. H.; Kwon, J. E.; Kim, J. H.; Olivier, Y.; Fischer, R.; Resel, R.; Gierschner, J.; Tada, H.; Park, S. Y., Highly Luminescent 2D-Type Slab Crystals Based on a Molecular Charge-Transfer Complex as Promising Organic Light-Emitting Transistor Materials. Adv Mater 2017, 29 (36).

5. Zambianchi, M.; Benvenuti, E.; Bettini, C.; Zanardi, C.; Seeber, R.; Gentili, D.; Cavallini, M.; Muccini, M.; Biondo, V.; Soldano, C.; Generali, G.; Toffanin, S.; Melucci, M., Anthracenebased molecular emitters for non-doped deep-blue organic light emitting transistors. Journal of Materials Chemistry C 2016, 4 (40), 9411-9417.

6. Orgiu, E.; Samori, P., 25th anniversary article: organic electronics marries photochromism: generation of multifunctional interfaces, materials, and devices. Adv Mater 2014, 26 (12), 1827 45 .

7. Chaudhry, M. U.; Muhieddine, K.; Wawrzinek, R.; Sobus, J.; Tandy, K.; Lo, S. C.; Namdas, E. B., Organic Light-Emitting Transistors: Advances and Perspectives. Advanced Functional Materials 2019, 30 (20). 
8. Wan, Y.; Deng, J.; Wu, W.; Zhou, J.; Niu, Q.; Li, H.; Yu, H.; Gu, C.; Ma, Y., Efficient Organic Light-Emitting Transistors Based on High-Quality Ambipolar Single Crystals. ACS Appl Mater Interfaces 2020, 12 (39), 43976-43983.

9. Samuel, I. D.; Turnbull, G. A., Organic semiconductor lasers. Chem Rev 2007, 107 (4), 1272-95.

10. Liu, D.; De, J.; Gao, H.; Ma, S.; Ou, Q.; Li, S.; Qin, Z.; Dong, H.; Liao, Q.; Xu, B.; Peng, Q.; Shuai, Z.; Tian, W.; Fu, H.; Zhang, X.; Zhen, Y.; Hu, W., Organic Laser Molecule with High Mobility, High Photoluminescence Quantum Yield, and Deep-Blue Lasing Characteristics. J Am Chem Soc 2020, 142 (13), 6332-6339.

11. Lee, S.; Lee, H. J.; Ji, Y.; Lee, K. H.; Hong, K., Electrochemiluminescent Transistors: A New Strategy toward Light-Emitting Switching Devices. Adv Mater 2020, e2005456.

12. Sun, Y.; Geng, H.; Peng, Q.; Shuai, Z., Computational Study on the Charge Transport and Optical Spectra of Anthracene Derivatives in Aggregates. Chemphyschem 2020, 21 (9), 952-957.

13. Wang, C.; Dong, H.; Hu, W.; Liu, Y.; Zhu, D., Semiconducting pi-conjugated systems in field-effect transistors: a material odyssey of organic electronics. Chem Rev 2012, 112 (4), 220867.

14. Kasha, M., Energy Transfer Mechanisms and the Molecular Exciton

Model for Molecular Aggregates. Radiation Research 1963, 20, 55-71.

15. Langevin, P., The recombination and mobilities of ions in gases. Ann Chim Phys 1903, 28, 433-530.

16. Fidder, H.; Knoester, J.; Wiersma, D. A., Superradiant Emission and Optical Dephasing in J-Aggregates. Chemical Physics Letters 1990, 171 (5-6), 529-536.

17. Liu, J.; Zhang, H.; Dong, H.; Meng, L.; Jiang, L.; Jiang, L.; Wang, Y.; Yu, J.; Sun, Y.; Hu, W.; Heeger, A. J., High mobility emissive organic semiconductor. Nat Commun 2015, 6, 10032.

18. Li, J.; Zhou, K.; Liu, J.; Zhen, Y.; Liu, L.; Zhang, J.; Dong, H.; Zhang, X.; Jiang, L.; Hu, W., Aromatic Extension at 2,6-Positions of Anthracene toward an Elegant Strategy for Organic Semiconductors with Efficient Charge Transport and Strong Solid State Emission. J Am Chem Soc 2017, 139 (48), 17261-17264.

19. Chuang, C.; Bennett, D. I. G.; Caram, J. R.; Aspuru-Guzik, A.; Bawendi, M. G.; Cao, J., Generalized Kasha's Model: T-Dependent Spectroscopy Reveals Short-Range Structures of 2D Excitonic Systems. Chem 2019, 5 (12), 3135-3150.

20. Hestand, N. J.; Spano, F. C., Expanded Theory of H- and J-Molecular Aggregates: The Effects of Vibronic Coupling and Intermolecular Charge Transfer. Chem Rev 2018, 118 (15), 70697163.

21. Spano, N. J. H. a. F. C., Molecular Aggregate Photophysics beyond the Kasha Model: Novel

Design Principles for Organic Materials. Accounts of chemical reasearch 2017.

22. Yamagata, H.; Pochas, C. M.; Spano, F. C., Designing J- and H-aggregates through wave function overlap engineering: applications to poly(3-hexylthiophene). J Phys Chem B 2012, 116 (49), 14494-503.

23. Song, J.; Gao, F.; Shi, B.; Liang, W., Identification of the mechanism of enhanced exciton interaction in rigidly linked naphthalene dimers. Phys Chem Chem Phys 2010, 12 (40), 13070-5.

24. Pan, F.; Gao, F.; Liang, W.; Zhao, Y., Nature of low-lying excited states in H-aggregated perylene bisimide dyes: results of TD-LRC-DFT and the mixed exciton model. $J$ Phys Chem $B$ 2009, 113 (44), 14581-7.

25. Hestand, N. J.; Spano, F. C., Interference between Coulombic and CT-mediated couplings 
in molecular aggregates: $\mathrm{H}$ - to J-aggregate transformation in perylene-based pi-stacks. $J$ Chem Phys 2015, 143 (24), 244707.

26. Subotnik, J. E.; Yeganeh, S.; Cave, R. J.; Ratner, M. A., Constructing diabatic states from adiabatic states: extending generalized Mulliken-Hush to multiple charge centers with boys localization. J Chem Phys 2008, 129 (24), 244101.

27. Head-Gordon, M.; Grana, A. M.; Maurice, D.; White, C. A., Analysis of Electronic Transitions as the Difference of Electron Attachment and Detachment Densities. The Journal of Physical Chemistry 1995, 99 (39), 14261-14270.

28. Kronik, L.; Stein, T.; Refaely-Abramson, S.; Baer, R., Excitation Gaps of Finite-Sized Systems from Optimally Tuned Range-Separated Hybrid Functionals. J Chem Theory Comput 2012, 8 (5), 1515-31.

29. Carter-Fenk, K.; Lao, K. U.; Liu, K. Y.; Herbert, J. M., Accurate and Efficient ab Initio Calculations for Supramolecular Complexes: Symmetry-Adapted Perturbation Theory with ManyBody Dispersion. $J$ Phys Chem Lett 2019, 10 (11), 2706-2714.

30. Shao, Y.; Gan, Z.; Epifanovsky, E.; Gilbert, A. T. B.; Wormit, M.; Kussmann, J.; Lange, A. W.; Behn, A.; Deng, J.; Feng, X.; Ghosh, D.; Goldey, M.; Horn, P. R.; Jacobson, L. D.; Kaliman, I.; Khaliullin, R. Z.; Kuś, T.; Landau, A.; Liu, J.; Proynov, E. I.; Rhee, Y. M.; Richard, R. M.; Rohrdanz, M. A.; Steele, R. P.; Sundstrom, E. J.; Woodcock, H. L.; Zimmerman, P. M.; Zuev, D.; Albrecht, B.; Alguire, E.; Austin, B.; Beran, G. J. O.; Bernard, Y. A.; Berquist, E.; Brandhorst, K.; Bravaya, K. B.; Brown, S. T.; Casanova, D.; Chang, C.-M.; Chen, Y.; Chien, S. H.; Closser, K. D.; Crittenden, D. L.; Diedenhofen, M.; DiStasio, R. A.; Do, H.; Dutoi, A. D.; Edgar, R. G.; Fatehi, S.; Fusti-Molnar, L.; Ghysels, A.; Golubeva-Zadorozhnaya, A.; Gomes, J.; Hanson-Heine, M. W. D.; Harbach, P. H. P.; Hauser, A. W.; Hohenstein, E. G.; Holden, Z. C.; Jagau, T.-C.; Ji, H.; Kaduk, B.; Khistyaev, K.; Kim, J.; Kim, J.; King, R. A.; Klunzinger, P.; Kosenkov, D.; Kowalczyk, T.; Krauter, C. M.; Lao, K. U.; Laurent, A. D.; Lawler, K. V.; Levchenko, S. V.; Lin, C. Y.; Liu, F.; Livshits, E.; Lochan, R. C.; Luenser, A.; Manohar, P.; Manzer, S. F.; Mao, S.-P.; Mardirossian, N.; Marenich, A. V.; Maurer, S. A.; Mayhall, N. J.; Neuscamman, E.; Oana, C. M.; Olivares-Amaya, R.; O'Neill, D. P.; Parkhill, J. A.; Perrine, T. M.; Peverati, R.; Prociuk, A.; Rehn, D. R.; Rosta, E.; Russ, N. J.; Sharada, S. M.; Sharma, S.; Small, D. W.; Sodt, A.; Stein, T.; Stück, D.; Su, Y.-C.; Thom, A. J. W.; Tsuchimochi, T.; Vanovschi, V.; Vogt, L.; Vydrov, O.; Wang, T.; Watson, M. A.; Wenzel, J.; White, A.; Williams, C. F.; Yang, J.; Yeganeh, S.; Yost, S. R.; You, Z.-Q.; Zhang, I. Y.; Zhang, X.; Zhao, Y.; Brooks, B. R.; Chan, G. K. L.; Chipman, D. M.; Cramer, C. J.; Goddard, W. A.; Gordon, M. S.; Hehre, W. J.; Klamt, A.; Schaefer, H. F.; Schmidt, M. W.; Sherrill, C. D.; Truhlar, D. G.; Warshel, A.; Xu, X.; Aspuru-Guzik, A.; Baer, R.; Bell, A. T.; Besley, N. A.; Chai, J.-D.; Dreuw, A.; Dunietz, B. D.; Furlani, T. R.; Gwaltney, S. R.; Hsu, C.-P.; Jung, Y.; Kong, J.; Lambrecht, D. S.; Liang, W.; Ochsenfeld, C.; Rassolov, V. A.; Slipchenko, L. V.; Subotnik, J. E.; Van Voorhis, T.; Herbert, J. M.; Krylov, A. I.; Gill, P. M. W.; Head-Gordon, M., Advances in molecular quantum chemistry contained in the Q-Chem 4 program package. Molecular Physics 2014, 113 (2), 184-215.

31. Sun, Q.; Berkelbach, T. C.; Blunt, N. S.; Booth, G. H.; Guo, S.; Li, Z.; Liu, J.; McClain, J. D.; Sayfutyarova, E. R.; Sharma, S.; Wouters, S.; Chan, G. K. L., PySCF: the Python-based simulations of chemistry framework. WIREs Computational Molecular Science 2017, 8 (1).

32. Stefan Dappricha, I. K. a., 2, K. Suzie Byuna, Keiji Morokumaa,U, Michael J. Frisch, A new ONIOM implementation in Gaussian98. Part I. The

calculation of energies, gradients, vibrational frequencies

and electric field derivatives. Journal of Molecular Structure (Theochem) 1999, 1-21.

33. Frisch, M. J.; Trucks, G. W.; Schlegel, H. B.; Scuseria, G. E.; Robb, M. A.; Cheeseman, J. 
R.; Scalmani, G.; Barone, V.; Petersson, G. A.; Nakatsuji, H.; Li, X.; Caricato, M.; Marenich, A. V.; Bloino, J.; Janesko, B. G.; Gomperts, R.; Mennucci, B.; Hratchian, H. P.; Ortiz, J. V.; Izmaylov, A. F.; Sonnenberg, J. L.; Williams; Ding, F.; Lipparini, F.; Egidi, F.; Goings, J.; Peng, B.; Petrone, A.; Henderson, T.; Ranasinghe, D.; Zakrzewski, V. G.; Gao, J.; Rega, N.; Zheng, G.; Liang, W.; Hada, M.; Ehara, M.; Toyota, K.; Fukuda, R.; Hasegawa, J.; Ishida, M.; Nakajima, T.; Honda, Y.; Kitao, O.; Nakai, H.; Vreven, T.; Throssell, K.; Montgomery Jr., J. A.; Peralta, J. E.; Ogliaro, F.; Bearpark, M. J.; Heyd, J. J.; Brothers, E. N.; Kudin, K. N.; Staroverov, V. N.; Keith, T. A.; Kobayashi, R.; Normand, J.; Raghavachari, K.; Rendell, A. P.; Burant, J. C.; Iyengar, S. S.; Tomasi, J.; Cossi, M.; Millam, J. M.; Klene, M.; Adamo, C.; Cammi, R.; Ochterski, J. W.; Martin, R. L.; Morokuma, K.; Farkas, O.; Foresman, J. B.; Fox, D. J. Gaussian 16 Rev. B.01, Wallingford, CT, 2016.

34. Varghese, S.; Park, S. K.; Casado, S.; Fischer, R. C.; Resel, R.; Milian-Medina, B.; Wannemacher, R.; Park, S. Y.; Gierschner, J., Stimulated Emission Properties of Sterically Modified Distyrylbenzene-Based H-Aggregate Single Crystals. J Phys Chem Lett 2013, 4 (10), 1597-602.

35. Katoh, R.; Suzuki, K.; Furube, A.; Kotani, M.; Tokumaru, K., Fluorescence Quantum Yield of Aromatic Hydrocarbon Crystals. J Phys Chem C 2009, 113 (7), 2961-2965.

36. Liu, J.; Zhu, W.; Zhou, K.; Wang, Z.; Zou, Y.; Meng, Q.; Li, J.; Zhen, Y.; Hu, W., Pyridylsubstituted anthracene derivatives with solid-state emission and charge transport properties. Journal of Materials Chemistry C 2016, 4 (16), 3621-3627.

37. Kazmaier, P. M.; Hoffmann, R., A Theoretical Study of Crystallochromy. Quantum Interference Effects in the Spectra of Perylene Pigments. Journal of the American Chemical Society 1994, $116(21), 9684-9691$.

\section{Supplementary Information for "Intermolecular Charge-Transfer- Induced Strong Optical Emission from Herringbone H-Aggregates"}

Qi Sun ${ }^{1}$, Jiajun Ren ${ }^{1}$, Tong Jiang ${ }^{1}$, Qian Peng ${ }^{2}, \mathrm{Qi} \mathrm{Ou}^{1 *}$ and Zhigang Shuai ${ }^{2 *}$

${ }^{I}$ MOE Key Laboratory of Organic OptoElectronics and Molecular Engineering, Department of Chemistry, Tsinghua University, Beijing 100084, P. R. China

${ }^{2}$ School of Chemical Sciences, University of Chinese Academy of Sciences, Beijing, 100049, P. R. China

Diagonalization of $\widehat{\boldsymbol{H}}$ in case of $\boldsymbol{t}_{\mathbf{h}} \approx-\boldsymbol{t}_{\mathbf{e}}=\boldsymbol{t}$. Corresponding eigenenergies and eigenstates of $\widehat{H}$ when $t_{\mathrm{h}} \approx-t_{\mathrm{e}}=t$ read,

$$
\begin{gathered}
E_{1}=E+J \\
E_{2}=E+\frac{s-J-\sqrt{(s+J)^{2}+8 t^{2}}}{2} \\
E_{3}=E+\frac{s-J+\sqrt{(s+J)^{2}+8 t^{2}}}{2} \\
\Psi_{2}=\frac{1}{\sqrt{C^{2}+1}}\left(C\left|\mathrm{FE}_{\mathrm{AS}}\right\rangle+\left|\mathrm{F}^{-} \mathrm{E}^{+}\right\rangle\right)
\end{gathered}
$$

${ }^{*}$ Corresponding authors. Email addresses: qiou@tsinghua.edu.cn; zgshuai@tsinghua.edu.cn. 


$$
\Psi_{3}=\frac{1}{\sqrt{C^{2}+1}}\left(-\left|\mathrm{FE}_{\mathrm{AS}}\right\rangle+C\left|\mathrm{~F}^{-} \mathrm{E}^{+}\right\rangle\right)
$$

where $s=E_{\mathrm{CT}}-E$, and $C=E_{\mathrm{CT}}-E-J+\sqrt{\left(E_{\mathrm{CT}}-E-J\right)^{2}+8 t^{2}}$. According to the expression of the eigenstates, one finds that CTE mainly couples with antisymmetric FE, while the symmetric bright FE remains unchanged. In such case, the lowest excited state of the $\mathrm{H}$-aggregates will be a mixing of $\left|\mathrm{F}^{-} \mathrm{E}^{+}\right\rangle$and $\left|\mathrm{FE}_{\mathrm{AS}}\right\rangle$, i.e., a transition dipole-forbidden state. CT-enhanced strong emission therefor will not occur in $\mathrm{H}$-aggregates when $t_{\mathrm{h}} \times t_{\mathrm{e}}<0$. If the transfer integral is relatively large, e.g., $|t|>\sqrt{\left|J^{2}+J s\right|}, E_{2}$ will become less than $E_{1}$ for J-aggregates, and the emission of J-aggregates will be quenched.

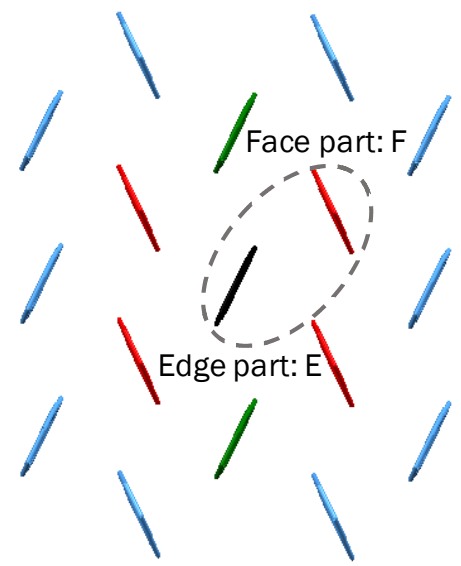

Figure S1. Herringbone crystal packing motif. Face-to-edge dimers correspond to the black-red molecule pair, and slipped $\pi-\pi$ dimers correspond to the black-green molecule pair. The "face" part and the "edge" part of the face-to-edge dimer in the ellipse are defined.
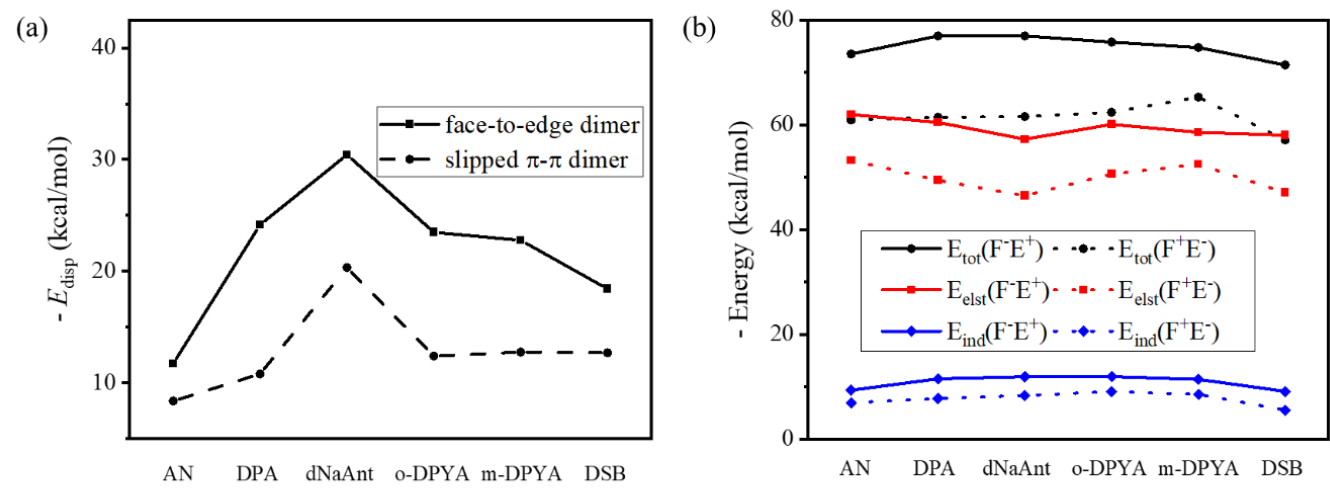

Figure S2. Intermolecular interactions in H-aggregate dimers. (a) The dispersion energies of face-toedge dimers and slipped $\pi-\pi$ dimers for all investigated systems at the level of CAM-B3LYP/def2-tzvpp. (b) XSAPT+MBD energy $\mathrm{E}_{\text {tot }}, \mathrm{E}_{\text {elst }}, \mathrm{E}_{\text {ind }}$ for two types of CTEs $\left(\mathrm{F}^{-} \mathrm{E}^{+}\right.$and $\left.\mathrm{F}^{+} \mathrm{E}^{-}\right)$of all the investigated faceto-edge dimers at the level of CAM-B3LYP/def2-tzvpp. 

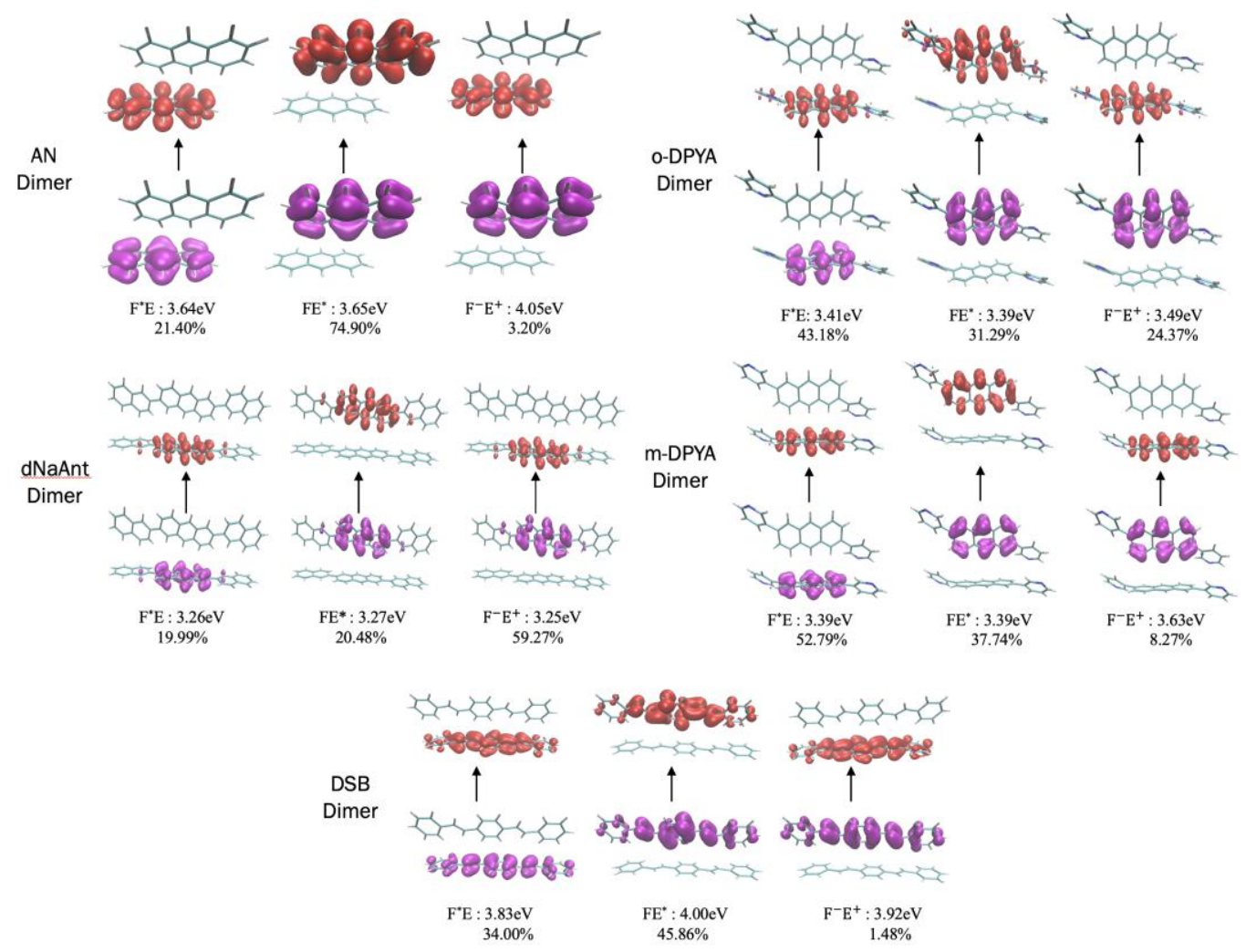

Figure S3. Attachment-detachment densities and excitation energies of the resulting diabatic states, $\mathrm{FE}^{*}, \mathrm{~F}^{*} \mathrm{E}$, and $\mathrm{F}^{-} \mathbf{E}^{+}$of AN, dNaAnt, o-DPYA, m-DPYA, and DSB given by Boys localized diabatization. The percentages listed below the excitation energy are the contribution of the corresponding diabatic state to the adiabatic $S_{1}$ state of each compounds. 


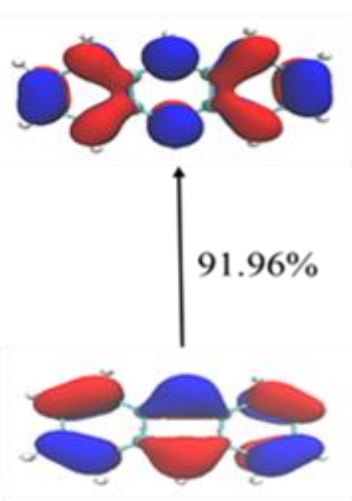

AN

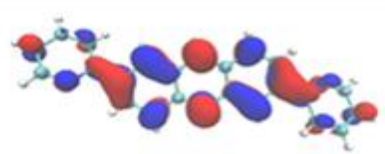

$\uparrow 95.36 \%$

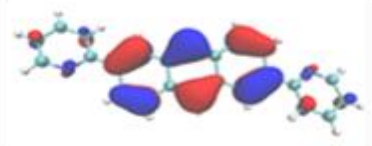

o-DPYA

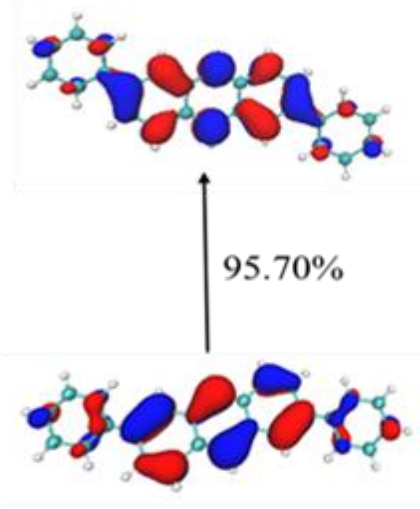

DPA

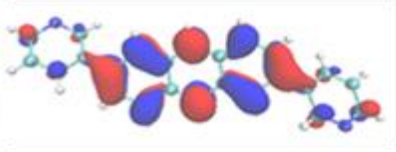

† $96.09 \%$

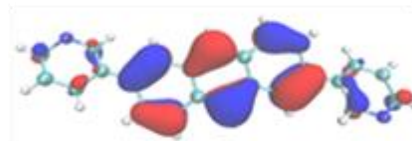

m-DPYA

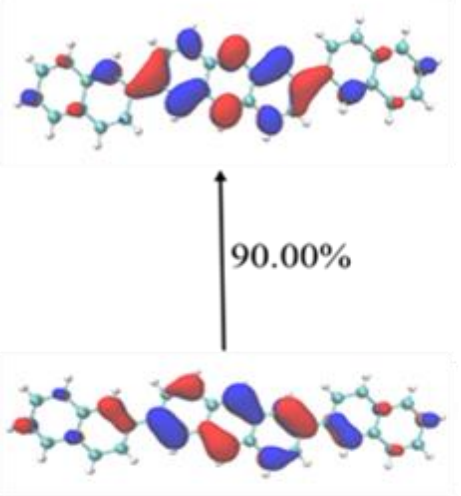

dNaAnt

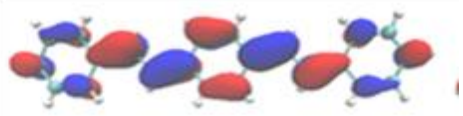

$\uparrow 91.84 \%$

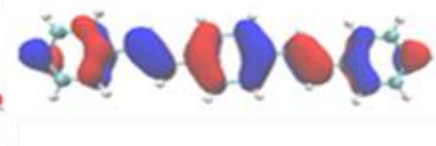

DSB

Figure S4. Visualized representation of the frontier orbitals (HOMO and LUMO) of the monomer for investigated compounds with the transition components of $S_{1}$ state.
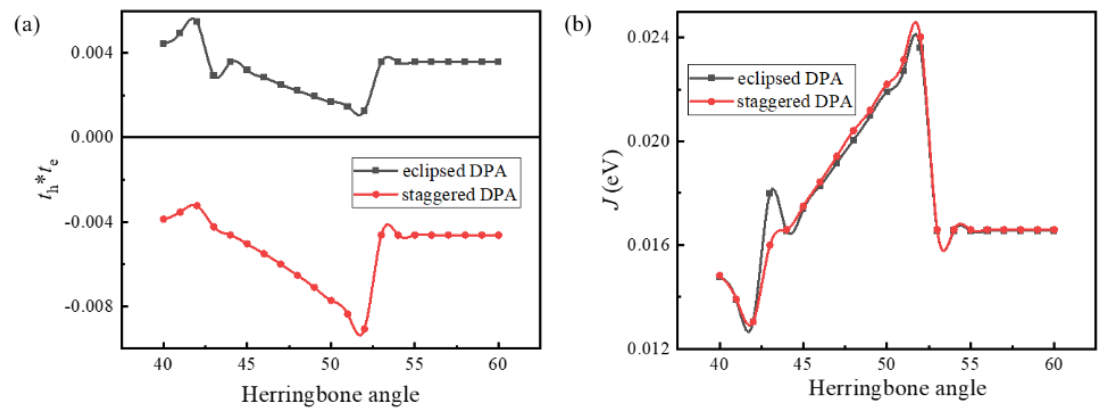

(c)

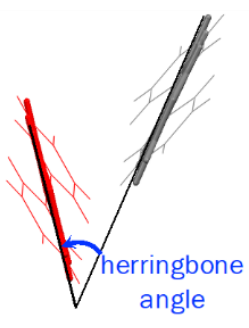

Figure S5. (a) Calculated $t_{\mathrm{h}} * t_{\mathrm{e}}\left(\mathrm{Unit}:(\mathrm{eV})^{2}\right)$ and (b) calculated $J$ (Unit: $\mathrm{eV}$ ) of the eclipsed and staggered DPA face-to-edge dimers with respect to the herringbone angles (Unit: ${ }^{\circ}$ ). The herringbone angle between the two monomers is defined in (c). 
(a)

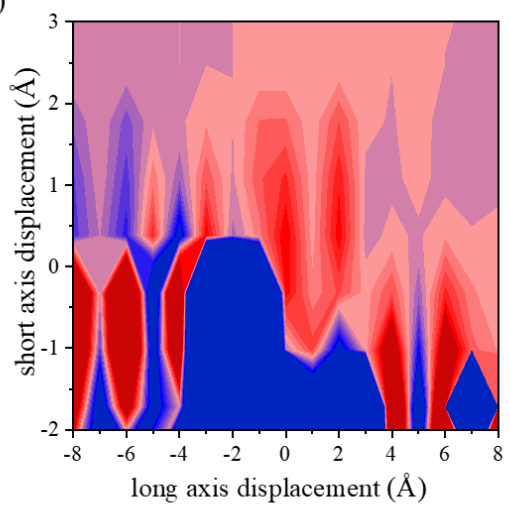

(c)

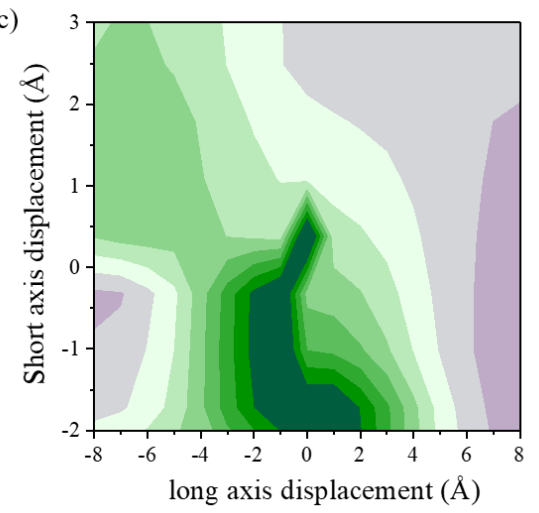

(b)
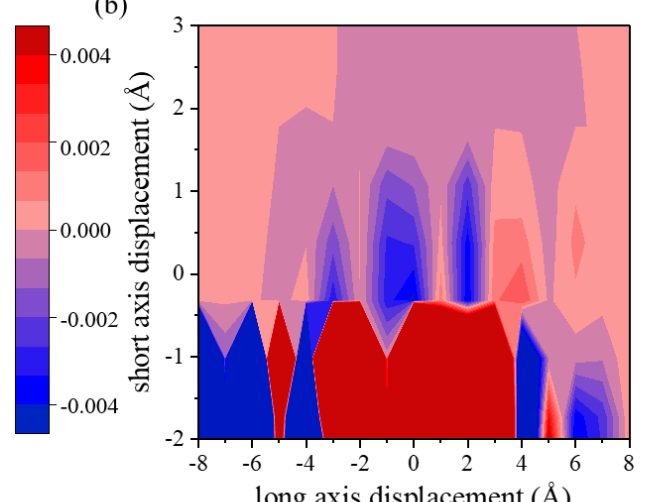

$-0.004$

$(\mathrm{d})$
-0.060
-

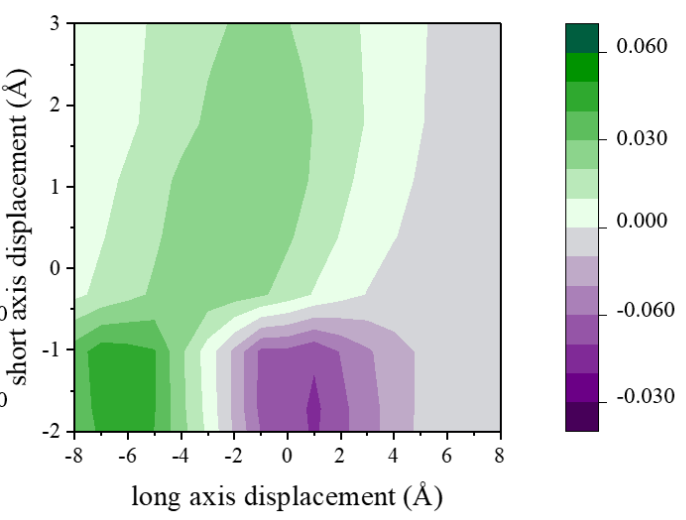

Figure S6. Calculated $t_{\mathrm{h}} * t_{\mathrm{e}}\left(\mathrm{Unit}:(\mathrm{eV})^{2}\right)$ and $J(U n i t: \mathrm{eV}) t_{\mathrm{h}} * t_{\mathrm{e}}$ with respect to the displacements along short and long axes of DPA face-to-edge dimer. (a) and (c) correspond to the calculated $t_{\mathrm{h}} * t_{\mathrm{e}}$ and $J$ of the eclipsed DPA dimer; (b) and (d) correspond to those of the staggered DPA dimer. 
Table S1. Centroid distance $d$ (Unit: $\AA$ ) of two types of dimers in the investigated HB aggregates and the optimal tuned $\omega$ values (Unit: Bohr ${ }^{-1}$ ). Exction coupling $J$ of DSB are in parenthesis (Unit: $\mathrm{meV})$.

\begin{tabular}{cccc}
\hline Compound & $d$ (face-to-edge dimers) & $d$ (slipped $\pi-\pi$ dimers) & $\omega$ \\
\hline An & 5.238 & 6.038 & 0.1868 \\
DPA & 4.823 & 6.245 & 0.1438 \\
dNaAnt & 4.822 & 5.980 & 0.1358 \\
o-DPYA & 4.816 & 6.235 & 0.1443 \\
m-DPYA & 4.860 & 6.280 & 0.1528 \\
DSB & $4.748(117)$ & $5.916(84)$ & 0.1564 \\
\hline
\end{tabular}

Table S2. XSAPT+MBD energy decomposition for two types of charge-transfer excitons of all investigated materials at the level of CAM-B3LYP/def2-tzvpp (in the unit of $\mathrm{kcal} / \mathrm{mol}$ )

\begin{tabular}{ccccccccccccccc}
\hline \multirow{2}{*}{ Compound } & \multicolumn{2}{c}{$E_{\text {tot }}$} & \multicolumn{2}{c}{$E_{\text {disp }}$} & \multicolumn{2}{c}{$E_{\text {elst }}$} & \multicolumn{2}{c}{$E_{\text {exch }}$} & \multicolumn{2}{c}{$E_{\text {ind }}$} & \multicolumn{2}{c}{$E_{\text {exch-Ind }}$} \\
& $\mathrm{F}^{-} E^{+}$ & $\mathrm{F}^{+} \mathrm{E}^{-}$ & $\mathrm{F}^{-}$ & $\mathrm{F}^{+} \mathrm{E}^{-}$ & $\mathrm{F}^{-} \mathrm{E}^{+}$ & $\mathrm{F}^{+} \mathrm{E}^{-}$ & $\mathrm{F}^{+}$ & $\mathrm{F}^{+} \mathrm{E}^{-}$ & $\mathrm{F}^{-} \mathrm{E}^{+}$ & $\mathrm{F}^{+} \mathrm{E}^{-}$ & $\mathrm{F}^{-} \mathrm{E}^{+}$ & $\mathrm{F}^{+} \mathrm{E}^{-}$ \\
\hline An & -73.52 & -60.91 & -11.54 & -12.00 & -61.95 & -53.22 & 7.86 & 9.06 & -9.32 & -6.93 & 1.45 & 2.17 \\
DPA & -76.96 & -61.43 & -24.06 & -24.37 & -60.47 & -49.41 & 15.94 & 16.63 & -11.50 & -7.74 & 3.13 & 3.25 \\
dNaAnt & -76.96 & -61.58 & -30.32 & -30.62 & -57.24 & -46.47 & 18.84 & 19.81 & -11.91 & -8.31 & 3.67 & 4.01 \\
o-DPYA & -75.80 & -62.38 & -23.38 & -23.66 & -60.08 & -50.59 & 15.98 & 16.88 & -11.94 & -9.06 & 3.63 & 4.05 \\
m-DPYA & -74.75 & -65.28 & -22.68 & -22.93 & -58.56 & -52.46 & 14.63 & 15.29 & -11.39 & -8.53 & 3.25 & 3.35 \\
DSB & -71.41 & -57.10 & -18.42 & -18.57 & -58.01 & -47.07 & 11.82 & 11.94 & -9.09 & -5.53 & 2.29 & 2.13 \\
\hline
\end{tabular}

Table S3. Three-state diabatic Hamiltonian (Unit: eV) and diabatic-to-adiabatic rotation matrix given by Boys localized diabatization method.

\begin{tabular}{|c|c|cc|}
\hline \multirow{2}{*}{ Compounds } & $\begin{array}{c}\text { Three-state diabatic } \\
\text { Hamiltonian }\end{array}$ & \multicolumn{3}{c|}{ Aiabatic-to-diabatic rotation } \\
& matrix \\
\hline \multirow{2}{*}{ DPA } & {$\left[\begin{array}{lll}3.321 & 0.012 & 0.076 \\
0.012 & 3.323 & 0.062 \\
0.076 & 0.062 & 3.375\end{array}\right] \quad\left[\begin{array}{ccc}0.627 & 0.481 & 0.608 \\
0.624 & -0.781 & -0.023 \\
-0.460 & -0.398 & 0.793\end{array}\right]$} \\
\hline
\end{tabular}




\begin{tabular}{|c|c|c|c|c|c|c|}
\hline \multirow{3}{*}{ An } & $\lceil 3.643$ & 0.008 & 0.0321 & $\lceil 0.463$ & 0.865 & 0.179 \\
\hline & 0.008 & 3.648 & 0.069 & 0.881 & -0.472 & 0.010 \\
\hline & 0.032 & 0.069 & $4.054]$ & 0.075 & 0.163 & 0.984 \\
\hline \multirow{3}{*}{$\mathrm{dNaAnt}$} & {$[3.259$} & 0.026 & $0.060]$ & {$[0.447$} & 0.453 & 0.7701 \\
\hline & 0.026 & 3.275 & 0.066 & 0.731 & -0.679 & 0.022 \\
\hline & 0.060 & 0.066 & $3.252]$ & 0.514 & 0.567 & 0.638 \\
\hline \multirow{3}{*}{ o-DPYA } & {$[3.413$} & 0.007 & 0.093 & 0.657 & -0.559 & 0.4947 \\
\hline & 0.007 & 3.389 & -0.036 & 0.540 & 0.810 & 0.215 \\
\hline & 0.093 & -0.036 & 3.492 & {$[-0.522$} & 0.118 & 0.842 \\
\hline \multirow{3}{*}{ m-DPYA } & [3.390 & 0.006 & 0.078 & {$[0.727$} & -0.614 & $0.288]$ \\
\hline & 0.006 & 3.388 & -0.041 & 0.629 & 0.772 & 0.075 \\
\hline & 0.078 & -0.041 & 3.631 & 0.267 & -0.122 & $0.955]$ \\
\hline \multirow{3}{*}{ DSB } & {$[3.829$} & 0.080 & $0.002]$ & {$[-0.583$} & 0.677 & 0.121 \\
\hline & 0.080 & 4.000 & 0.020 & 0.621 & 0.307 & 0.703 \\
\hline & L0.002 & $\underline{0}$ & 3.916 & -0.520 & -0.452 & 0.700 \\
\hline
\end{tabular}

Note that the diabatic Hamiltonian in Table S3 are given in the basis of $\left\{\left|\mathrm{F}^{*} \mathrm{E}\right\rangle ;\left|\mathrm{FE}^{*}\right\rangle ;\left|\mathrm{F}^{-} \mathrm{E}^{+}\right\rangle\right\}$, which is of the following form:

$$
\widehat{H}=\left[\begin{array}{ccc}
E & J & t_{\mathrm{h}} \\
J & E & t_{\mathrm{e}} \\
t_{\mathrm{h}} & t_{\mathrm{e}} & E_{\mathrm{CT}}
\end{array}\right]
$$

The excitation energies of the locally excited states and the CTE $\left(\mathrm{F}^{-} \mathrm{E}^{+}\right)$can be read from the diagonal elements of $\widehat{H}$, and the exciton coupling $(J)$ as well as the charge and hole transfer integrals $\left(t_{\mathrm{e}}\right.$ and $\left.t_{\mathrm{h}}\right)$ can be read from the off-diagonal elements of $\widehat{H}$. The diabatic-to-adiabatic unitary rotation matrix $\mathbf{U}$ connects the adiabatic states and the obtained localized diabatic states $\left\{\left|\mathrm{F}^{*} \mathrm{E}\right\rangle ;\left|\mathrm{FE}^{*}\right\rangle ;\left|\mathrm{F}^{-} \mathrm{E}^{+}\right\rangle\right\}$via

$$
\left[\begin{array}{l}
\Psi_{S_{1}} \\
\Psi_{S_{2}} \\
\Psi_{S_{3}}
\end{array}\right]=\mathbf{U}\left[\begin{array}{c}
\Xi_{\mathrm{F}^{*} \mathrm{E}} \\
\Xi_{\mathrm{FE}^{*}} \\
\Xi_{\mathrm{F}^{-} \mathrm{E}^{+}}
\end{array}\right]
$$

By plugging in the matrix elements of $\mathbf{U}$, the adiabatic excited states of the dimer can be written as a linear combination of $\mathrm{F}^{*} \mathrm{E}, \mathrm{FE}^{*}$, and $\mathrm{F}^{-} \mathrm{E}^{+}$. If the coefficients of $\mathrm{F}^{*} \mathrm{E}$ and $\mathrm{FE}^{*}$ are of the same sign, then that adiabatic state is formed via the coupling between the CTE and the symmetric FE, otherwise it is formed via the coupling between the CTE and the antisymmetric FE. The contribution of each diabatic state to a given adiabatic state can be obtained via the square of the coefficients of each diabatic state. These contributions of $\mathrm{S}_{1}$ for each compound are listed below the corresponding attachment-detachment densities.

Table S4. Calculated excitation energy of the first singlet states $\left(E_{S_{1}}\right.$, unit in eV) and corresponding transition dipole moments $\left(\mu_{S_{1}}\right.$, unit in Debye) of the isolated dimer, dimer in solid phase via QM/MM approach and the isolated monomer at the level of $\omega \mathrm{B} 97 \mathrm{X}-\mathrm{D} * / 6-31 \mathrm{G}(\mathrm{d})$.

\begin{tabular}{cccccccc} 
& \multicolumn{2}{c}{ Dimer } & \multicolumn{2}{c}{ Dimer $(\mathbf{Q M} / \mathbf{M M})$} & \multicolumn{2}{c}{ Monomer } \\
\cline { 2 - 7 } Compound & $E_{\mathrm{S}_{1}}$ & $\mu_{\mathrm{S}_{1}}$ & $E_{\mathrm{S}_{1}}$ & $\mu_{\mathrm{S}_{1}}$ & $E_{\mathrm{S}_{1}}$ & $\mu_{\mathrm{S}_{1}}$ \\
\hline An & 3.62 & 3.00 & 3.63 & 3.10 & 3.65 & 2.51 \\
DPA & 3.20 & 3.47 & 3.25 & 3.83 & 3.28 & 3.76 \\
\hline
\end{tabular}




\begin{tabular}{ccccccc}
\hline dNaAnt & 3.17 & 4.51 & 3.19 & 5.37 & 3.28 & 6.36 \\
& & & & & & \\
o-DPYA & 3.33 & 1.64 & 3.27 & 1.80 & 3.41 & 3.25 \\
m-DPYA & 3.34 & 1.81 & 3.35 & 1.82 & 3.40 & 2.96 \\
DSB & 3.68 & 2.37 & 3.48 & 0.44 & 3.79 & 11.40 \\
\hline
\end{tabular}

Table S5. Exciton couplings and transfer integrals computed via FMO method for all investigated systems. Numbers are shown in the unit of meV.

\begin{tabular}{cccc}
\hline Compound & $J$ & $t_{\mathrm{h}}$ & $t_{\mathrm{e}}$ \\
\hline An & 4 & 27 & 73 \\
DPA & 9 & 68 & 58 \\
dNaAnt & 39 & 32 & 62 \\
o-DPYA & 8 & 97 & -43 \\
m-DPYA & 6 & 83 & -45 \\
DSB & 117 & 3 & 48 \\
\hline
\end{tabular}

